\title{
Effects of Catalysts on Dielectric Properties and D. C. Conduction in Poly(ethylene terephthalate)
}

\author{
Hiroyuki Sasabe, ${ }^{*}$ Kiwa Sawamura, ${ }^{* *}$ Shogo Saito, ${ }^{*}$ \\ and Kentaro YODA***
}

(Received March 1, 1971)

\begin{abstract}
Dielectric relaxations and d.c. conduction in poly(ethylene terephthalate)(PET) were studied from the point of view of how types and concentration of catalysts used in polymerization affect these electrical properties. D.C. conductivity in PET is affected considerably by the use of catalysts; that is, residual catalysts themselves are ionized in the polymer and contribute to d.c. conduction. On the other hand, from the evidence that the dielectric $\alpha$-relaxation process is scarcely affected by catalysts but that the dielectric $\beta$-relaxation process is separated into two modes by the use of catalysts, it is concluded that the molecular structure of PET is scarcely affected by catalysts as a whole, but might be changed in local structures, such as chain ends. The molecular mechanisms of the $\beta$-relaxation in PET are attributable to the local oscillations of frozen-in main chains and the hindered rotations of the terminal carboxyl groups.
\end{abstract}

KEY WORDS Poly(ethylene terephthalate)/Dielectric Relaxation / D.C. Conduction / Catalyst / Ionic Conduction/Local Mode Relaxation /

Studies on electrical properties in organic polymers have been vigorously carried out for the past twenty years, but still left unsolved the problem of current carriers in electrical conduction. The current carriers in many polymers have been supposed to be ions, judging from the negative pressure dependence of d.c. conductivity. ${ }^{1}$ Such is also the case with conduction in poly(ethylene terephthalate). The problem that must then be solved is the source of the ionic impurities. As a source of ionic impurities, organic and/or inorganic impurities involved in raw materials, products from thermal decomposition of polymer and residual catalysts can be considered. Effects of these impurities on electrical properties in polymers have been studied in connection with the molecular structure of the polymers. However quantitative treatments on the relationship between species and concentration of catalysts and electrical

* Electrotechnical Laboratory, Tanashi, Tokyo, Japan.

** Mitsubishi Paper Mills Ltd., Marunouchi, Tokyo, Japan.

*** Katata Research Institute, Toyobo Co. Ltd., Otsu, Shiga, Japan. properties of polymers have so far rarely been carried out. The main objectives of this paper are focussed on this point.

Effects of catalysts are classified into two types: (1) polymer structures, e.g., terminal groups, molecular weight, and stereoregularity, are changed depending upon species of catalysts used, then electrical properties of the polymer are also changed (indirect effect), and (2) residual catalysts themselves are ionized in the polymer and contribute to d.c. conduction. Thus the electrical properties of the polymer are affected (direct effect). These electrical properties depend on temperature. The expected effects of catalysts on electrical properties are shown in Table I.

In this paper we will investigate dielectric $\alpha$ and $\beta$ relaxation processes and d.c. conduction in poly(ethylene terephthalate) samples with different catalysts used in polymerization as a function of temperature, and discuss which effect is expected, direct or indirect, in the electrical properties of the polymer.

\section{EXPERIMENTAL}

Five samples of poly(ethylene terephthalate) 
Effects of Catalysts on Electrical Properties in PET

Table I. Expected effects of catalysts on electrical properties of polymers

\begin{tabular}{lcll}
\hline Electrical properties & $\begin{array}{c}\text { Temperature } \\
\text { region }\end{array}$ & Molecular mechanism & $\begin{array}{c}\text { Effects of catalysts } \\
\text { (expected) }\end{array}$ \\
\hline Dielectric relaxation & $T>T_{\mathrm{g}}$ & $\begin{array}{l}\alpha \text {-relaxation (micro-Brownian motion of } \\
\text { amorphous main chains) } \\
\beta \text {-relaxation (local oscillation of frozen- } \\
\text { in main chains) }\end{array}$ & Indirect \\
\hline D.C. conduction & $T<T_{\mathrm{g}}$ & $\begin{array}{l}\text { Ionic conduction } \\
\text { Inic conduction }\end{array}$ & Direct (+indirect) \\
\hline
\end{tabular}

Table II. Characteristics of samples

\begin{tabular}{|c|c|c|c|c|c|c|}
\hline Sample & Catalyzer & $M_{n}$ & $\begin{array}{c}\mathrm{DEG} / \mathrm{EG} \\
\mathrm{mol} \%\end{array}$ & $\begin{array}{l}\chi, \\
\%\end{array}$ & $\mathrm{~g} / \mathrm{cm}^{3} \stackrel{\rho,}{\text { at }} 21^{\circ} \mathrm{C}$ & ${ }^{{ }^{\circ} \mathrm{C}}$ \\
\hline PET-0 & ? (commercial grade) & 17280 & 2.4 & 74.9 & 1.346 & \\
\hline PET-I & none & 13060 & 3.6 & 15.5 & 1.344 & 65.2 \\
\hline PET-II & 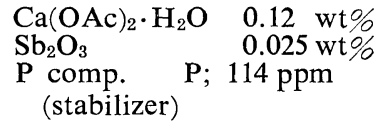 & 19390 & - & 33.9 & 1.344 & 68.9 \\
\hline PET-III & $\mathrm{Zn}(\mathrm{OAc})_{2} 2 \cdot \mathrm{H}_{2} \mathrm{O} \quad 0.036 \mathrm{wt} \%$ & 23420 & 2.7 & 50.4 & 1.343 & 68.5 \\
\hline PET-IV & $?$ & 23040 & - & 70.4 & 1.343 & 67.5 \\
\hline
\end{tabular}

(PET) were used. Characteristics of these samples are listed in Table II. Here $\bar{M}_{n}$ is the numberaveraged molecular weight and calculated from the relation, ${ }^{2}$

$$
[\eta]=(3.0 \pm 1.2) \times 10^{-4} \bar{M}_{n}^{0.77 \pm 0.09}
$$

where $[\eta]$ is the intrinsic viscosity and estimated from the viscosity $\eta$ of the phenol-tetrachloroethane solution at $30^{\circ} \mathrm{C}$. Application of eq 1 to the present study requires the identity in the distribution of molecular weights among samples. There would exist some differences in the distribution among samples as polymerized. However, all samples used for measurements of [ $\eta]$ as well as electrical properties had been heated up to the melt and then would have the most probable distribution $\left(M_{w} / M_{n}=2 ; M_{w}\right.$, the weight-averaged molecular weight) due to the exchange reactions of ester linkages in the melt. Therefore eq 1 would be applicable to the present system. Chain segments of PET generally contain not only ethylene glycol groups(EG) but small amounts of diethylene glycol group(DEG). Mole fraction of DEG in the main chains, DEG/EG, is determined from the gas chromatography method, using poly(ethylene glycol) (PEG 20M) column with temperature programmed from 100 to $200^{\circ} \mathrm{C}$ at rate of $4^{\circ} \mathrm{C} / \mathrm{min}$. Concentration of $\mathrm{COOH}$ groups in $\mathrm{PET},[\mathrm{COOH}]$, is determined from the titration with $\mathrm{NaOH}(0.1 N)$-benzyl alcohol mixture. Mole fraction of terminal $\mathrm{COOH}$ groups, $\chi$, is calculated from the relation,

$$
\begin{aligned}
\chi= & \frac{[\mathrm{COOH}]}{[\mathrm{OH}]+[\mathrm{COOH}]} \times 100=\frac{[\mathrm{COOH}]}{2 / \bar{M}_{n}} \\
& \times 10^{-6} \times 100(\%)
\end{aligned}
$$

$\rho$ is the density at $21^{\circ} \mathrm{C}$, and determined from the density gradient tube of $n$-heptane-tetrachlorocarbon system. $T_{\mathrm{g}}$ is the glass transition temperature at 1 atm and estimated from the break point in $\log \sigma v s$. temperature plot, where $\sigma$ is the d.c. conductivity. PET-I was prepared from terephthalic acid and ethylene glycol by direct esterification followed by a polycondensation without catalyst at $240^{\circ} \mathrm{C}$ under reduced pressure. PET-0 is a commercial grade sample and in sheet form of $0.5-\mathrm{mm}$ thickness. Other samples were made into thin films of $0.2-\mathrm{mm}$ thickness by compression moulding at $255^{\circ} \mathrm{C}$ ( $T_{\mathrm{m}}$, about $250^{\circ} \mathrm{C}$ ), and then quenching in an icewater mixture. These films were nearly amorphous (degree of crystallinity is about $8 \%$ ). The three terminals method was adopted for electrical measurements, and measurements of d.c. con- 
ductivity and dielectric losses at low frequencies of the polymer were thus made possible. Electrodes were prepared by deposition of silver in vacuo. Complex dielectric constant $\varepsilon^{*}\left(=\varepsilon^{\prime}-\right.$ $\left.i \varepsilon^{\prime \prime}\right)$ was measured in the range of frequency from $10^{-5}$ to $10^{6} \mathrm{~Hz}$, and of temperature from -100 to $+100^{\circ} \mathrm{C}$. D.C. conductivity $\sigma$ was measured in the temperature range above room temperature. $\sigma$ was calculated from the steady state (leakage) current after absorption current was vanished. Since absorption (charging) current in PET could be superposed on the inverted discharging current in this temperature region, this current would be due to the dielectric $\alpha$ relaxation process in PET. Details of electrical measurements were described in the previous papers. ${ }^{1,3}$

\section{RESULTS AND DISCUSSION}

\section{D.C. Conduction}

Figure 1 shows the relationship between d.c. conductivity $\sigma$ and temperature $T$ for the five samples. Values of $\mathrm{d}(\log \sigma) / \mathrm{d} T$ change suddenly in the vicinity of $70^{\circ} \mathrm{C}$ for all samples. These breaks correspond to the glass transition temperature $T_{\mathrm{g}}$, as discussed in previous papers. ${ }^{1,4} \sigma$ in the glassy state $\left(T<T_{\mathrm{g}}\right)$ is less than $10^{-17}$ $\mathrm{ohm}^{-1} \mathrm{~cm}^{-1}$, which is the limit of the accuracy of measurement. On the other hand, $\sigma$ in the liquid state $\left(T>T_{\mathrm{g}}\right)$ increases markedly with increases in $T$, and the accuracy of measurement is sufficiently high. As seen from Figure 1, $\sigma$ for PET-I, $\sigma$ (PET-I), is clearly less than those for the other four samples. This fact suggests that residual catalysts in the polymer can be ionized and become a source of charge carriers. $\sigma$ (PET-II) is slightly larger than $\sigma$ (PET-III). This implies that the sample polymerized with a large quantity of catalysts becomes more conductive, though not only the quantity but the degree of ionization of the catalyst should be considered. Figure 2 shows the relationship between $\log \sigma$ and $\boldsymbol{P}$ for PET-I and PET-II. The break in this plot corresponds to the glass transition point. For both samples $\mathrm{d}(\log \sigma) / \mathrm{d} P$ is negative above and below the glass transition point. Consequently, we can conclude that ionic conduction is dominant in PET-II. Conduction in PET-I should be also ionic, but

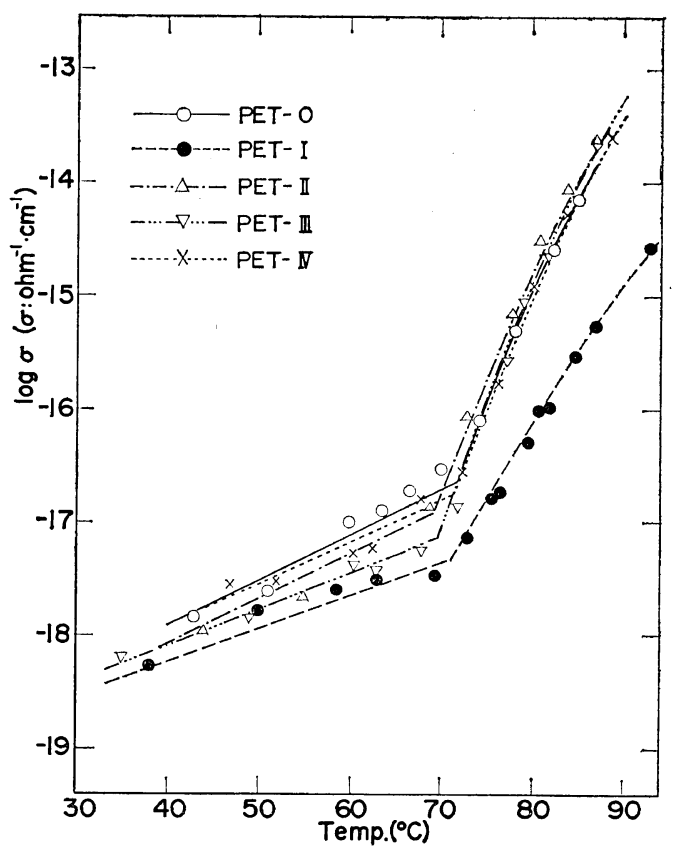

Figure 1. Temperature dependence of d.c. conductivity for five samples.

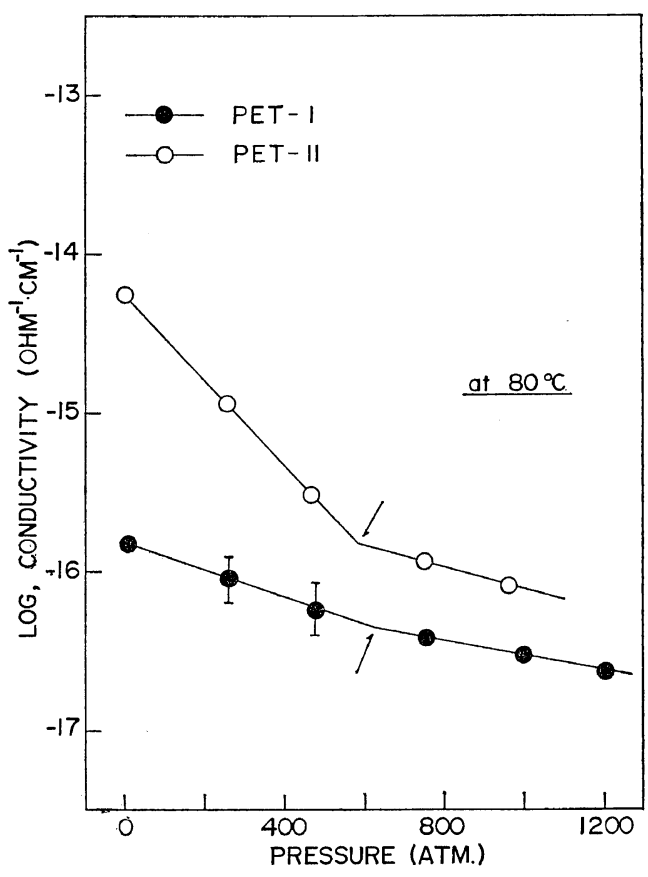

Figure 2. Pressure dependence of d.c. conductivity for PET-I and PET-II. Arrows indicate the glass transition points. 
further investigations are needed.

In order to clarify the mechanism of d.c. conduction in the polymer, we studied the effects of crystallization on d.c. conduction. Crystallization of samples PET-I and PET-II were carried out in silicone-oil bath at $110^{\circ} \mathrm{C}$ for $1.5 \mathrm{hr}$. Degree of crystallinity $X$ is calculated from density $\rho$ as

$$
X=\frac{\rho_{\mathrm{c}}}{\rho} \frac{\rho-{ }_{\mathrm{a}}}{\rho_{\mathrm{c}}-\rho_{\mathrm{a}}} \times 100(\%)
$$

where $\rho_{\mathrm{c}}$ and $\rho_{\mathrm{a}}$ are the density of crystalline and amorphous states respectively. Values of $\rho_{\mathrm{c}}$ and $\rho_{\mathrm{a}}$ are calculated from the molecular packing as 1.455 and $1.335 \mathrm{gcm}^{-3}$, respectively. $X$ for PET-I is equal to $40.5 \%$ and $X$ for PETII to $40.0 \%$. As the crystallization temperature is low, numerous small spherulites are generated in PET samples. As the reproducibility of data on $\varepsilon^{\prime \prime} v s . \log f$ and $\sigma$ at various temperatures is very good, the crystallization of the samples during measurements is negligible. Figure 3 shows the plots of $\log \sigma$ against $1 / T$ for amor-

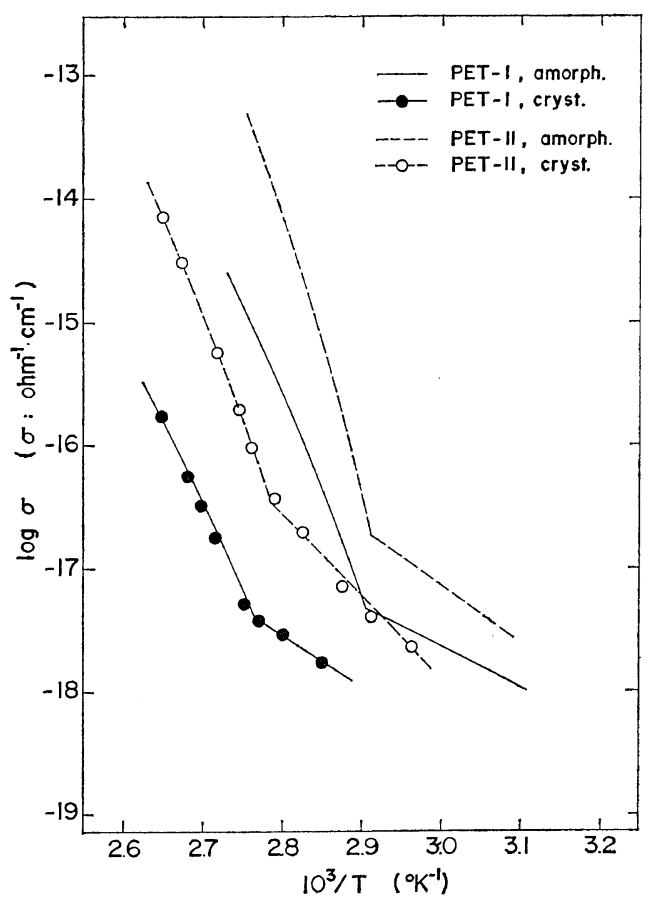

Figure 3. Plots of $\log \sigma$ against $1 / T$ for amorphous and crystallized samples. phous and crystallized samples. Breaks are observed in these plots. As the break on $\log \sigma$ vs. temperature plot corresponds to the glass transition, the shift of the break point means variation of $T_{\mathrm{g}}$ with crystallization. An interesting fact is that the value of $\sigma$ at the break point is scarcely changed due to polymer crystallization. This suggests that the concentration of ionic carriers at $T_{\mathrm{g}}$ is almost independent of crystallization, because the glass transition can be regarded as a reference point for ionic transportation in polymers. The value of $\sigma$ at $T_{\mathrm{g}}$ for four samples are in the following order

$$
\begin{aligned}
\sigma(\text { PET-I, amorph. }) & \simeq \sigma(\text { PET-I, cryst. }) \\
\ll \sigma(\text { PET-II, amorph. }) & \simeq \sigma(\text { PET-II, cryst. })
\end{aligned}
$$

This result is considered to be a manifestation of the difference in concentration of ionic carriers. From the comparison of $\sigma$ for crystallized samples with $\sigma$ for amorphous one at a given temperature above $T_{\mathrm{g}}$, it is found that $\sigma$ decreases remarkably with crystallization. Judging from the fact that $\sigma$ at $T_{\mathrm{g}}$ is almost independent of crystallization, this result is mainly attributable to reduction in carrier mobility: Ambolski's proposal that conducting ions are partly trapped or bound in the crystalline region would be unacceptable.

Temperature dependence of $\sigma$ above $T_{\mathrm{g}}$ is expressed by the WLF equation,

$$
\log \frac{\sigma(T)}{\sigma\left(T_{\mathrm{g}}\right)}=\frac{C_{1}\left(T-T_{\mathrm{g}}\right)}{C_{2}+\left(T-T_{\mathrm{g}}\right)}
$$

Parameters $C_{1}$ and $C_{2}$ for amorphous PET-I and PET-II samples were given as

PET-I, amorph.; $C_{1}=24.39, \quad C_{2}=167.3$

PET-II, amorph.; $C_{1}=13.33, C_{2}=55.3$.

Values of WLF parameters calculated from the temperature dependence of dielectric relaxation time are $C_{1}=19.61$ and $C_{2}=40.5$ for both samples, which is discussed in the next section. In the case of PET-II, $C_{2}$ for the $\sigma-T$ relation is nearly equal to $C_{2}$ for the $\tau-T$ relation. This fact means that the temperature dependence of $\sigma$ is governed exclusively by that of the ionic mobility, though $\sigma$ is the linear function of the carrier (ionic) concentration and the carrier (ionic) mobility. In such a case, the ratio of $C_{1}$ 
for the $\sigma-T$ relation to $C_{1}$ for the $\tau-T$ relation corresponds to the ratio of critical hole size for the ionic transport to that for the segmental motion in the polymer. ${ }^{6}$ In the case of PET-I, on the other hand, $C_{2}$ for the $\sigma-T$ relation is definitely different from $C_{2}$ for the $\tau-T$ relation. If the ionic mobility $\mu$ is separately determined from $\sigma$, the $\mu-T$ relation is also of the WLF type, and $C_{2}$ for the $\mu-T$ relation may be equal to that for the $\tau-T$ relation. Hence the temperature dependence of $\sigma$ in PET-I is determined not only by that of the ionic mobility, but by that of concentration of the free ions. Therefore any information on the hole size for the ionic transport cannot be obtained from the parameter $C_{1}$.

Let us now use the rate process theory for analysis of the $\sigma-T$ data. From the relationship between $\log \sigma$ and $1 / T$ which somewhat deviates from a linear relation, one can determine the average value of apparent activation energy $\Delta H^{*}$. Values of $\Delta H^{*}$ for samples are listed in Table III. It was found that $\Delta H^{*}$ for PET-II is larger than $\Delta H^{*}$ for PET-I, regardless whether the samples are amorphous or crystallized. The apparent activation energy $\Delta H^{*}$ is the sum of the activation energies for the ionic transport $\left(\Delta H^{*}(\mu)\right)$ and that for the ionization $\left(\Delta H^{*}(n)\right)$ of low-molecular-weight molecules introduced from raw materials, thermal decomposition of polymer, residual catalysts, and so on. The difference in $\Delta H^{*}(n)$ between two samples, PETI and PET-II, would be so small that the difference in $\Delta H^{*}$ would lead to the conclusion that $\Delta H^{*}(\mu)$ in PET-II is larger than $\Delta H^{*}(\mu)$ in PET-I. This implies that the carrier size in PET-I is smaller than that in PET-II. ${ }^{1}$

A similar conclusion can be directly obtained from the pressure dependence of $\sigma$ which is shown in Figure 2: activation volume $\Delta V^{*}$ obtained from $\mathrm{d}(\log \sigma) / \mathrm{d} P$ for PET-I is definitely smaller than that for PET-II. If the difference in activation volume for ionization between PET-I and PET-II is ignored, the larger $\Delta V^{*}$ corresponds to the larger volume of transporting ions. This evidence supports ionic conduction being the dominant mechanism for electrical conduction in PET-I, but the source of charge carriers in PET-I is not clear as yet.

\section{Dielectric $\alpha$-Relaxation Process}

The dielectric $\alpha$-relaxation process in PET has

Table III. Values of activation energy for amorphous and crystallized samples

\begin{tabular}{lc}
\hline Sample & $\Delta H^{*}, \mathrm{kcal} \mathrm{mol}^{-1}$ \\
\hline PET-I, amorph. & 74 \\
PET-I, cryst. & 64 \\
PET-II, amorph. & 94 \\
PET-II, cryst. & 77 \\
\hline
\end{tabular}

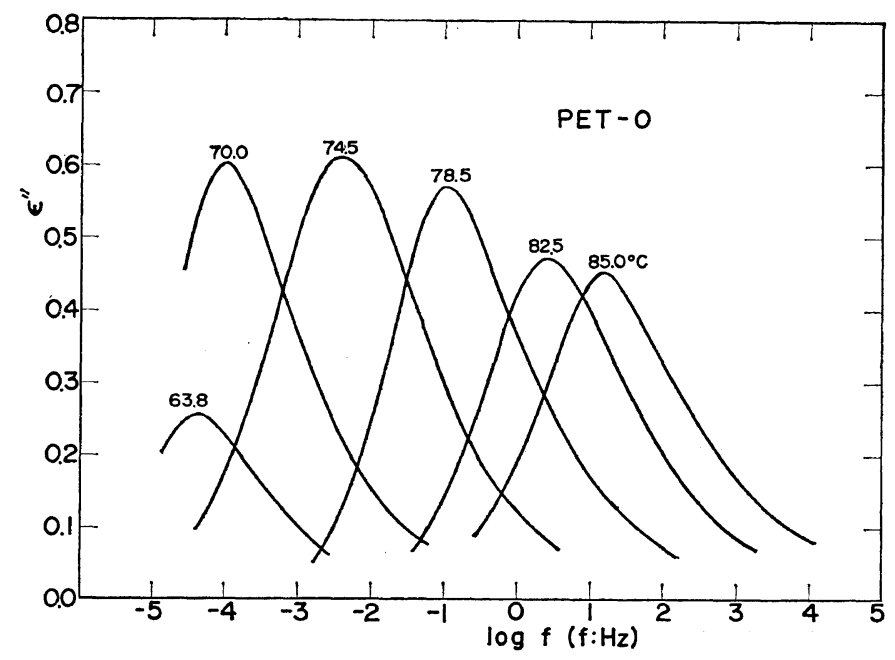

Figure 4. Dielectric absorption curves at various fixed temperatures for PET-0 ( $\alpha$-relaxation process). 
Effects of Catalysts on Electrical Properties in PET

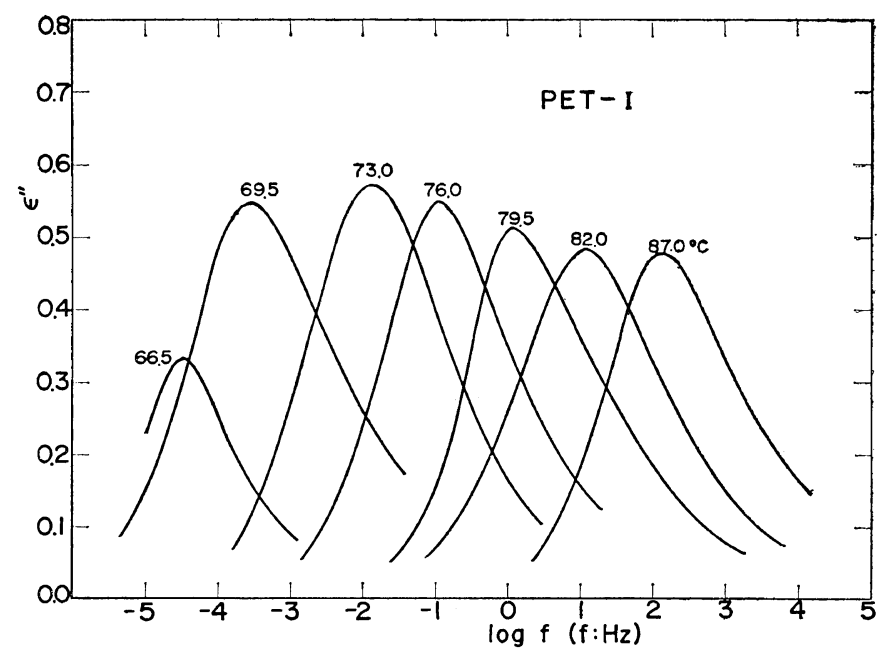

Figure 5. Dielectric absorption curves at various fixed temperatures for PET-I ( $\alpha$-relaxation process).

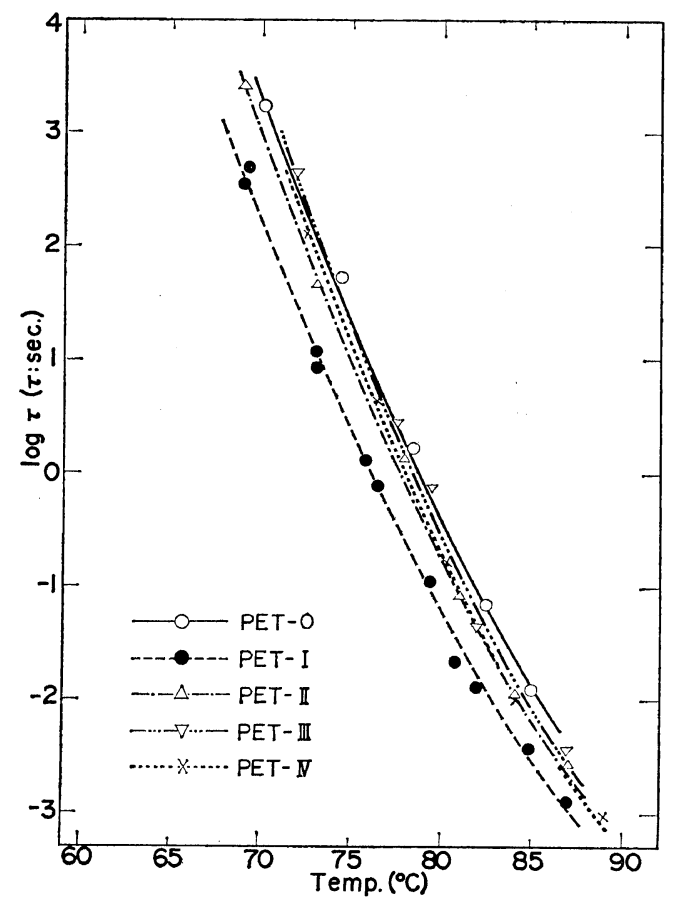

Figure 6. Temperature dependence of average dielectric relaxation time for five samples.

been closely studied and is interpreted as being due to the micro-Brownian motion of amorphous main chains. Average relaxation time $\bar{\tau}$ is determined from $f_{\max }$ as $\bar{\tau}=1 / 2 \pi f_{\max }{ }^{\prime}$ where $f_{\max }$ is the frequency at which the dielectric absorption curve ( $\varepsilon^{\prime \prime}$ vs. $\log f$ plots) becomes a maximum. Figures 4 and 5 show $\varepsilon^{\prime \prime}$ vs. $\log f$ plots at various fixed temperatures for PET-0 and PET-I, respectively. Temperature of $\bar{\tau}$ for five samples are shown in Figure 6. There are little differences among the curves for PET-0, -II, -III, and -IV; only the curve for PET-I is different from other curves. However all curves are of WLF type, and parameters $C_{1}$ and $C_{2}$ are independent of the samples, as listed in Table IV. $T_{\mathrm{g}}$ of PET-I is smaller than that of other samples by about three degrees. This corresponds to the fact that the molecular weight of PET-I is the smallest of the five samples.

Figure 7 shows the temperature dependence of dielectric increment for the $\alpha$-relaxation $\Delta \varepsilon_{\alpha}$ $\left(=\varepsilon_{0}-\varepsilon_{\infty}\right) . \Delta \varepsilon_{\alpha}$ was calculated from the dielectric absorption curve graphically, using the relation,

$$
\Delta \varepsilon=\frac{4.6}{\pi} \int_{-\infty}^{+\infty} \varepsilon^{\prime \prime} \mathrm{d}(\log f)
$$

There is no difference in the five samples within the limit of experimental error. Hence it is reasonable to consider that the catalyst scarcely affects the molecular structure of PET. This is more conspicuous if one compares the shape of the $\alpha$-absorption curves. Figure 8 shows the reduced loss $\left(\varepsilon^{\prime \prime} / \varepsilon^{\prime \prime}{ }_{\max }\right)$ curves as a function of $\log \left(f / f_{\max }\right)$ for five samples. In the distribution of $\tau$ no differences are observed among the samples. Distribution function of $\tau, F(\ln \tau)$, 
H. Sasabe, K. Sawamura, S. Saito, and K. Yoda

Table IV. WLF parameters for samples

\begin{tabular}{lccccc}
\hline Sample & $C_{1}$ & $C_{2}$ & $T_{\mathrm{g}},{ }^{\circ} \mathrm{C}$ & $\tau\left(T_{\mathrm{g}}\right), \mathrm{sec}$ & $T_{\mathrm{g}}{ }^{\mathrm{a}},{ }^{\circ} \mathrm{C}$ \\
\hline PET-I & 19.61 & 40.5 & 67.0 & $3.2 \times 10^{3}$ & 65 \\
PET-0, -II, -III, -IV & 19.61 & 40.5 & 69.5 & $3.2 \times 10^{3}$ & 68 \\
\hline
\end{tabular}

a Determined from DSC measurement.

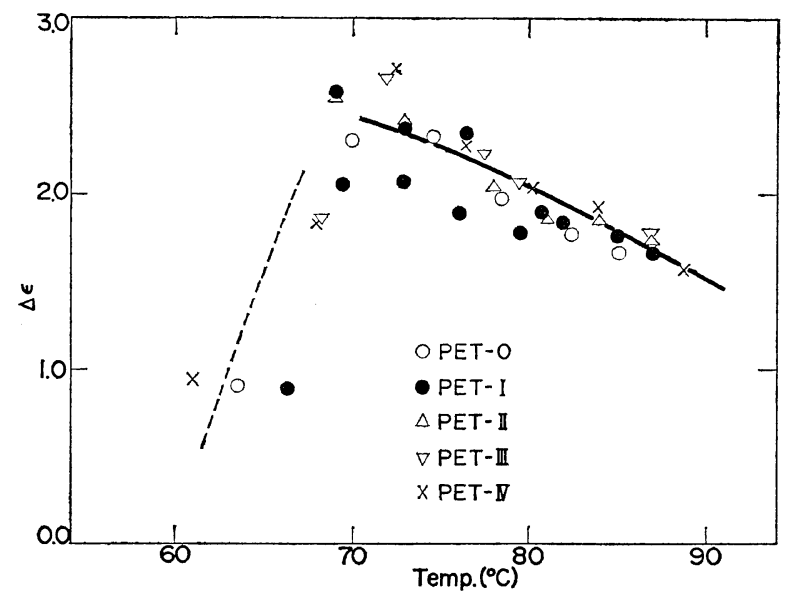

Figure 7. Temperature dependence of dielectric increment for $\alpha$-relaxation process.

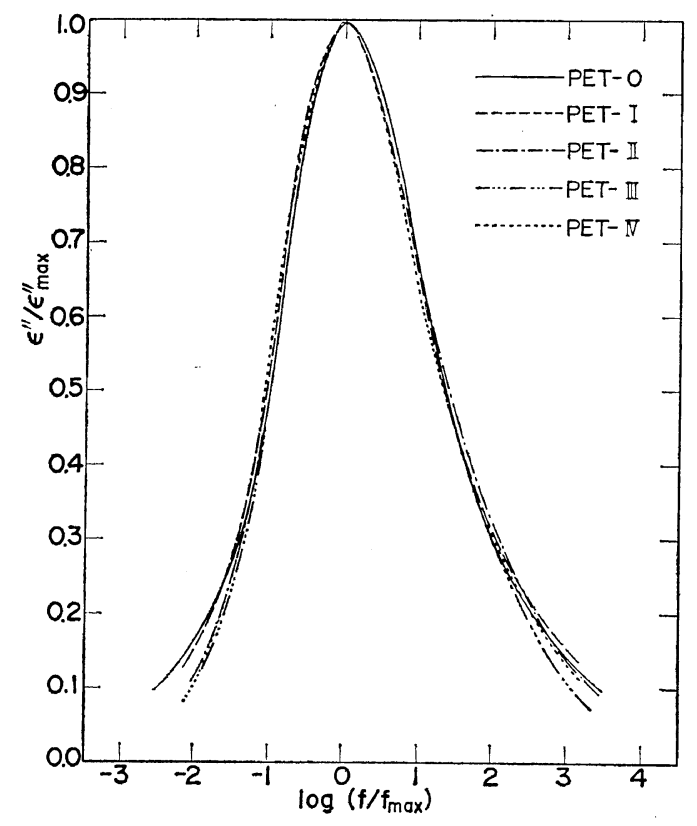

Figure 8. Reduced loss curves as a function of reduced frequency for five samples. can be determined from the reduced curve of $\varepsilon^{\prime \prime} / \varepsilon^{\prime \prime} \max$ vs. $\log \left(f / f_{\max }\right)$, applying the ColeCole theory. As seen from Figure $8, F(\ln \tau)$ is slightly asymmetric with respect to the average time $\bar{\tau}$, and given as

$$
\begin{aligned}
F(s) & =\frac{1}{2 \pi} \frac{\sin 0.7 \pi}{\cosh 0.7 s+\cos 0.7 \pi} ; \quad \tau>\bar{\tau} \\
& =\frac{1}{2 \pi} \frac{\sin 0.55 \pi}{\cosh 0.55 s+\cos 0.55 \pi} ; \quad \tau<\bar{\tau}
\end{aligned}
$$

where $s=\ln \tau / \bar{\tau}$. This distribution of $\tau$ is slightly narrower than the distribution obtained by Yamafuji and Ishida for PET. ${ }^{7}$ This is because the reduced curve in Figure 8 is obtained from the lower frequency data so that it is not affected by the superposition of the $\beta$-absorption in the higher frequency side.

Consequently, from the data of the $\alpha$-absorption, we can conclude that catalysts scarcely affect the molecular structure of PET as a whole.

\section{Dielectric $\beta$-Relaxation Process}

$\beta$-relaxation process in PET is located in the vicinity of $-50^{\circ} \mathrm{C}$ and $1 \mathrm{~Hz}$ in the mechanical 
or dielectric absorption curves. The molecular mechanism of this process has been interpreted as being due to the local oscillations of frozenin main chains. A detailed mechanical study by Illers and Breuer, ${ }^{8}$ however, revealed that the $\beta$-relaxation peak in PET is not a single peak but a double one. This behavior was observed as an asymmetry of the loss curve, $G^{\prime \prime}$ vs. $T$, and was better indicated by the plot of $\left(\partial G^{\prime} / \partial \log f\right)_{T}$ against $T$, where $G^{\prime}$ and $G^{\prime \prime}$ are the real and imaginary parts of the modulus, respectively. We have also observed double peaks in the dielectric absorption curves, $\varepsilon^{\prime \prime} v s$. $\log f$ or $\varepsilon^{\prime \prime} v s$. $T$, for the $\beta$-relaxation process. Double peaks are better resolved in the frequency dependence of $\varepsilon^{\prime \prime}$ at fixed temperatures than in the temperature dependence of $\varepsilon^{\prime \prime}$ at fixed frequencies. Figure 9 shows an example of $\varepsilon^{\prime \prime} v s$.

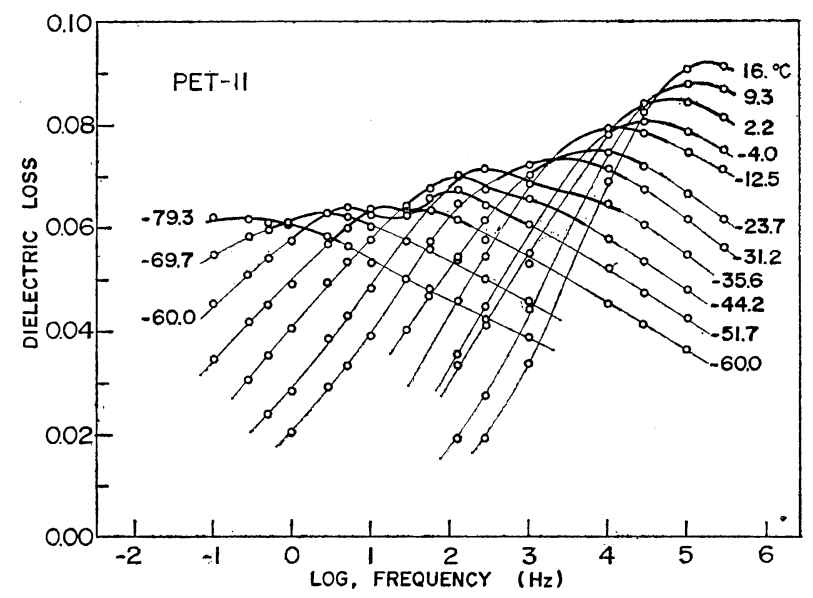

Figure 9. Dielectric absorption curves at various fixed temperatures for PET-II ( $\beta$-relaxation process).

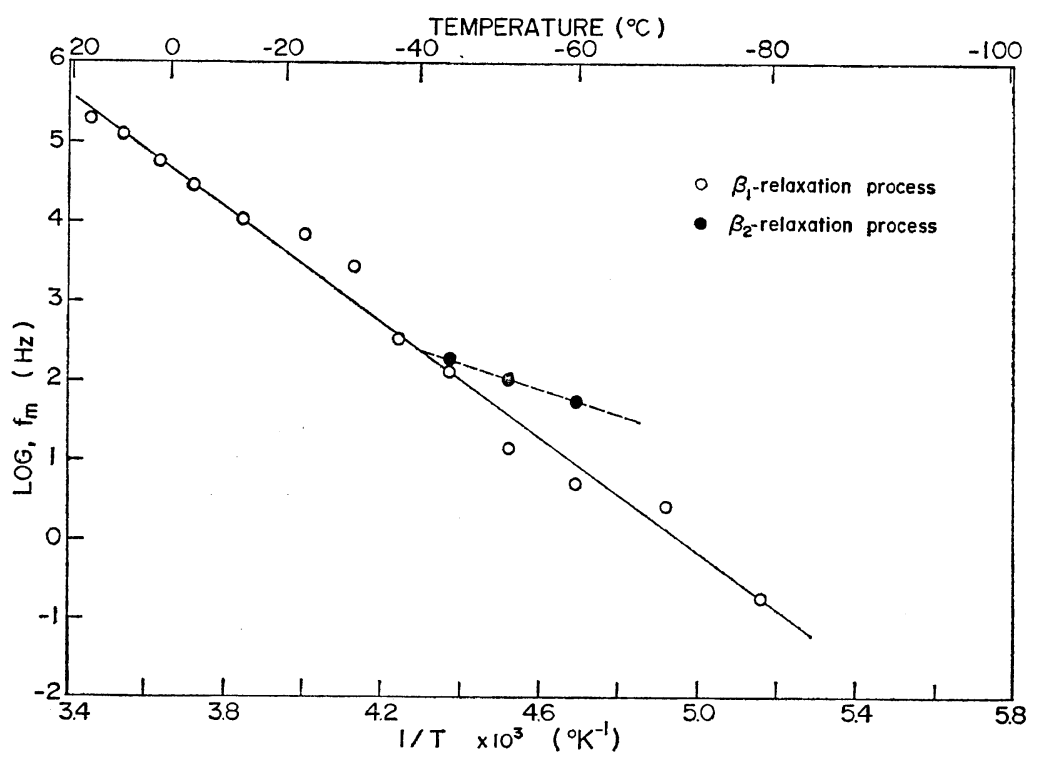

Figure 10. The frequency-temperature location of the maximum of the $\beta$ relaxation in PET-II. 


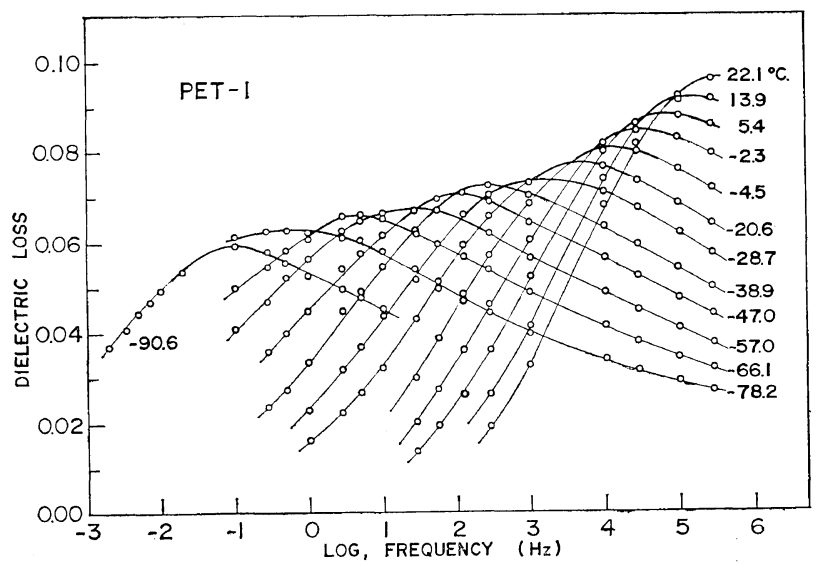

Figure 11. Dielectric absorption curves at various fixed temperatures for PET-I ( $\beta$-relaxation process).

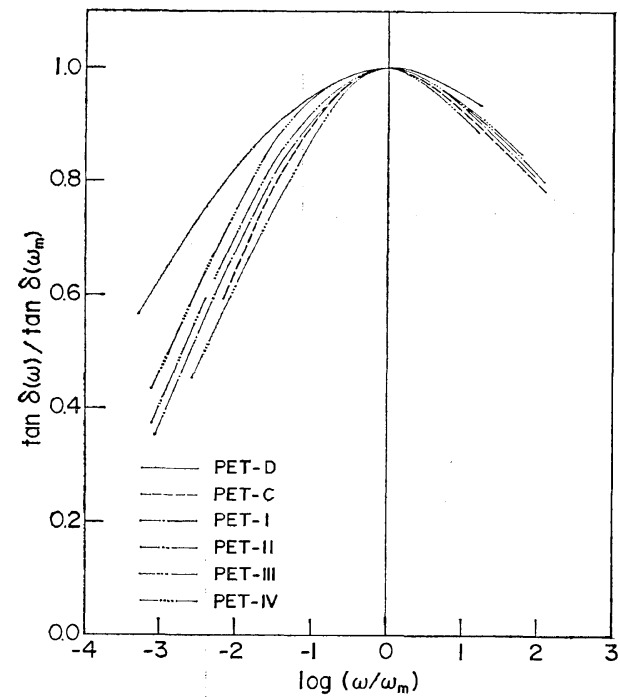

Figure 12. Reduced loss curves as a function of reduced frequency for various samples at $-30^{\circ} \mathrm{C}$.

$\log f$ plots for PET-III. At room temperature the absorption curve has a single peak. However with decreases in temperature, a shoulder appeared in the high frequency side of the absorption curve. This shoulder grows gradually to become a double peak with decreases in temperature. The high-frequency-side peak, however, disappears in the very low temperature range, because the relaxation strength for the corresponding molecular motion would decrease definitely with decreases in temperature in this range. Figure 10 shows the frequency-temperature location of the maximum of the dielectric $\beta$-relaxation in PET-II. Double peak can be commonly in the PET-0, -II, -III, -IV, -C, and -D samples. Here PET-C is a crystalline sample of PET-0 crystallized at $112^{\circ} \mathrm{C}$ for $30 \mathrm{hr}$ and PET-D is a drawn sample of PET-0 stretched at $65^{\circ} \mathrm{C}$ to $500-\%$ elongation. Therefore the $\beta$ process in PET would be attributed to two (or more) kinds of molecular motions.

Figure 11 shows the $\beta$-absorption curves for PET-I. A conspicuous difference between the absorption curve for PET-I and that for other samples is that PET-I shows single peaks only over the whole temperature and frequency ranges studied. The reason why a resolution of peak is not observed in PET-I will be discussed later. Figure 12 shows reduced absorption curves, $\tan \delta(\omega) / \tan \delta\left(\omega_{\mathrm{m}}\right) \quad v s . \quad \log \left(\omega / \omega_{\mathrm{m}}\right)$, for various samples at $-30^{\circ} \mathrm{C}$. Here $\omega$ is an angular frequency $(=2 \pi f)$ and $\tan \delta$ is a loss factor $\left(=\varepsilon^{\prime \prime} /\right.$ $\left.\varepsilon^{\prime}\right)$. As seen from this figure, there are little differences among distributions of relaxation times for the $\beta$ process in PET-I, -II, -III, and -IV. Therefore $\beta$-relaxation process in PET-I would be also attributable to two molecular motions. The one relaxation process located in the lower-frequency side of the $\beta$ process is designated here as the $\beta_{1}$-relaxation process, and the other as the $\beta_{2}$-relaxation process. Figure 12 indicates that the distribution in PET-C is rather narrow but that in PET-D is very broad. 
The following three models may be considered for the molecular mechanism of the $\beta$-relaxation: (1) local oscillation of $\mathrm{COO}$ groups involved in the frozen-in main chains (local mode relaxation), (2) local motion of DEG groups in the main chains, and (3) rotational motion of terminal $\mathrm{COOH}$ groups. Illers and Breuer have proposed that the model (1) is composed of two modes; one is due to trans isomers (corresponding to the $\beta_{1}$-relaxation) and the other is due to gauche isomers (corresponding to the $\beta_{2}$-relaxation). According to the recent work done mechanically by Kawai, et al. ${ }^{9}$ the secondary relaxation processes were separated into three kinds of submechanism; Mode I and Mode II are related not only to the hindrance potentials in the neighborhood of the parallelized and unparallelized phenyl groups (as postulated by Illers and Breuer) but also to the hindered rotations of methylene sequences, and Mode III is related to the localized hindered rotations of methylene sequences. The last mode, however, appeared in far lower-temperature regions than the $\beta$ relaxation process, and hence this is omitted here. Mode I and Mode II correspond to the $\beta_{1}$ - and the $\beta_{2}$-relaxations, respectively. From these interpretations, however, the following facts obtained in the present study are inexplicable; a resolution of the $\beta$-relaxation peak is not observed in PET-I and there is a large difference between the activation energy for the $\beta_{1}$ relaxation $\left(\simeq 17 \mathrm{kcal} \mathrm{mol}^{-1}\right.$; calculated from Figure 10) and that for the $\beta_{2}$-relaxation $(\simeq 11$ $\mathrm{kcal} \mathrm{mol}^{-1}$ ), and the distribution of the relaxation times for the PET-D sample is the broadest despite the fact that PET-D is considered to be richest in trans configuration by elongation. The model (2) is disqualified because double peaks are not clearly observed in PET-I, which contains more DEG groups than PET-IV (Table II).

According to Yamafuji and Ishida, ${ }^{7}$ the dielectric increment due to model (1), $\Delta \varepsilon_{1}$, is given as

$$
\Delta \varepsilon_{1}=\frac{4 \pi}{3}\left(E_{\mathrm{r}} / E\right)\left(N_{1} \mu_{1}^{2} / G^{\prime} a N_{1}^{-2 / 3}\right)
$$

where $E_{\mathrm{r}} / E$ is the ratio of Onsager's internal field to the applied field, $N_{1}$ is the number of dipoles per unit volume, $\mu_{1}$ is the effective dipole moment of COO group, $a$ is the effective radius of the motional unit, and $G^{\prime}$ is the dynamic shear modulus. These parameters are evaluated as $N_{1}=2 \times 4.09 \times 10^{21} \quad\left(\rho=1.31 \mathrm{~g} \mathrm{~cm}^{-3}\right.$ at $\left.0^{\circ} \mathrm{C}\right), \mu_{1}=1.6 \mathrm{D}, E_{\mathrm{r}} / E=3.23\left(\varepsilon_{0}=3.7\right.$ and $\varepsilon_{\infty}=$ 3.3), $a=4 \times 10^{-8} \mathrm{~cm}, G^{\prime}=9 \times 10^{9}$ dyne $\mathrm{cm}^{-2}$ (at 1 $\mathrm{Hz}$ and $0^{\circ} \mathrm{C}$ ), if we choose the motional unit as the half of the monomer unit. Then $\Delta \varepsilon_{1}$ is estimated as 0.28. As $G^{\prime}$ decreases with increases in temperature, $\Delta \varepsilon_{1}$ increases with temperature. Figure 13 shows the temperature dependence of $\Delta \varepsilon_{\beta}$ for the $\beta$-relaxation process in various samples. $\Delta \varepsilon_{\beta}$ is estimated graphically as well as $\Delta \varepsilon_{\alpha} . \Delta \varepsilon_{\beta}$ for PET-0 is considerably smaller than $\Delta \varepsilon_{\beta}$ for other samples. The reason for this is unknown, but it might be that only PET-0 is an as-received sample (commercial grade) and may be rolled. $\Delta \varepsilon_{\beta}$ is slightly larger than $\Delta \varepsilon_{1}$, but increases with temperature as well as $\Delta \varepsilon_{1}$.

Rotational motion of terminal $\mathrm{COOH}$ groups in the glassy state should be treated as a hindered rotation in a potential field formed by surrounding molecules, and the 'site' model is a good expression for this molecular motion. According to Ishida and Yamafuji, ${ }^{10}$ contribution of the dipole orientations caused by the transi-

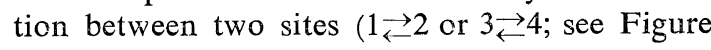
14) to the dielectric increment, $\Delta \varepsilon_{3}$, due to model (3) are given by the equation,

$$
\begin{aligned}
\Delta \varepsilon_{3}= & \frac{4 \pi N_{3} \mu_{3}^{2}}{3 k T}\left(\frac{3 \varepsilon_{0}}{2 \varepsilon_{0}+\varepsilon_{\infty}}\right)\left(\frac{\varepsilon_{\infty}+2}{3}\right)^{2} 4 W_{0}\left[\frac{\gamma}{1+\gamma}\right. \\
& \left.+\frac{\rho \gamma}{1+\rho \gamma}\right] \sin ^{2} \Theta_{0}
\end{aligned}
$$

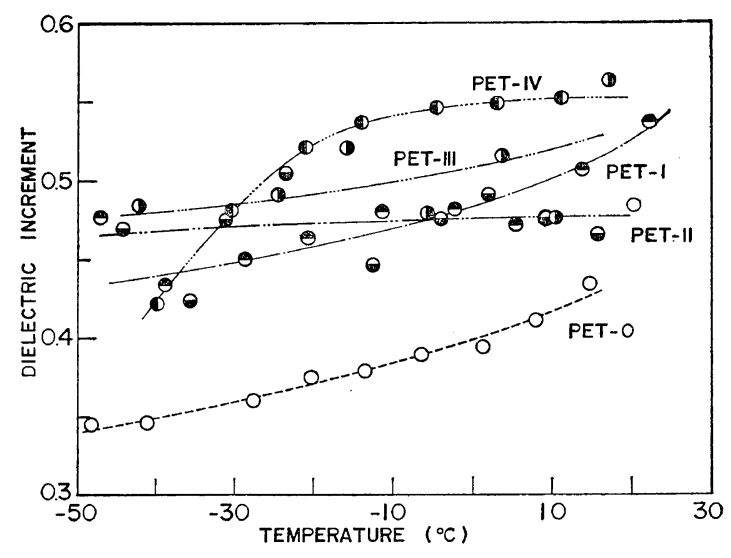

Figure 13. Temperature dependence of dielectric increment for the $\beta$-relaxation process in various samples. 


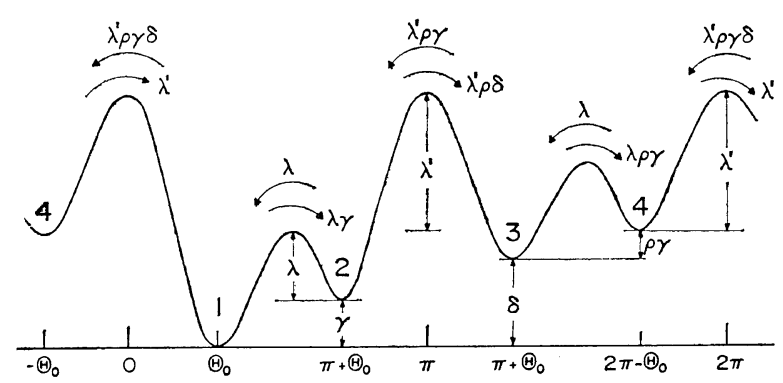

Figure 14. Schematic diagram for the transition probabilities among the sites (after Ishida and Yamafuji ${ }^{10}$ ).

Table V. Values of $N_{3}, \varepsilon_{\max }^{\prime \prime}, \varepsilon_{0}, \varepsilon_{\infty}$, and $\Delta \varepsilon_{3}{ }^{\prime}$ at $0^{\circ} \mathrm{C}$

\begin{tabular}{lccccc}
\hline Sample & $N_{3}$ (calcd) & $\varepsilon_{\max }^{\prime \prime}($ obsd $)$ & $\varepsilon_{0}$ (obsd) & $\varepsilon_{\infty}$ (obsd) & $\Delta \varepsilon_{3}^{\prime}$ (calcd) \\
\hline PET-0 & $6.81 \times 10^{19}$ & 0.077 & 3.60 & 3.27 & 0.13 \\
PET-I & $1.87 \times 10^{19}$ & 0.066 & 3.70 & 3.33 & 0.04 \\
PET-II & $2.75 \times 10^{19}$ & 0.052 & 3.66 & 3.30 & 0.05 \\
PET-III & $5.21 \times 10^{19}$ & 0.060 & 3.61 & 3.24 & 0.10 \\
PET-IU & $3.38 \times 10^{19}$ & 0.050 & 3.69 & 3.26 & 0.07 \\
\hline
\end{tabular}

where $N_{3}$ is the number of the terminal carboxyl groups per unit volume, $\mu_{3}$ is the effective dipole in the transition between two sites $(1 \rightleftarrows 2$ or $3 \rightleftarrows 4)$ and $\gamma, \delta$, and $\rho \gamma$ are the quantities whose values are between 0 and 1 . In the low-temperature regions below $T_{\mathrm{g}}, \gamma, \delta$, and $\rho \gamma$ are respectively smaller than unity, but they should approach unity near $T_{\mathrm{g}}$. Thus $\Delta \varepsilon_{3}$ increases with temperature, because the temperature dependence of $\Delta \varepsilon_{3}$ is strongly governed by that of $\gamma$ and $\rho \gamma$. If $\gamma, \delta$, and $\rho \gamma$ reach unity, free rotation of the dipole may be possible. Hence the dielectric increment $\Delta \varepsilon_{3}{ }^{\prime}$ for the free rotation of terminal $\mathrm{COOH}$ groups would give the upper limit of $\Delta \varepsilon_{3}$. Therefore, we adopt the free rotation model instead of model (3) for the order estimation of $\Delta \varepsilon_{3} . \Delta \varepsilon_{3}{ }^{\prime}$ is given by the Debye equation,

$$
\Delta \varepsilon_{3}{ }^{\prime}=\frac{4 \pi}{27 k T}\left(\varepsilon_{0}+2\right)\left(\varepsilon_{\infty}+2\right) N_{3} \mu_{3}{ }^{2}
$$

Values of $\varepsilon_{0}$ and $\varepsilon_{\infty}$ for the samples were respectively estimated from the Cole-Cole plot (Table V). Assuming $\mu_{3}=2.32 \mathrm{D}$, values of $\Delta \varepsilon_{3}{ }^{\prime}$ for various samples are calculated as shown in Table V. They increase slightly with an increase in $N_{3}$, but are 0.13 at most. $\Delta \varepsilon_{3}$ is about one-

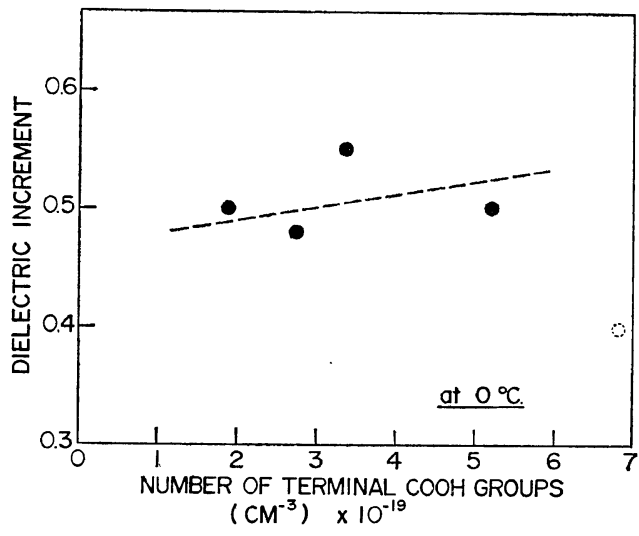

Figure 15. Relationship between number of terminal carboxyl groups per unit volume and observed values of dielectric increment for the $\beta$-relaxation process at $0^{\circ} \mathrm{C}$.

third of $\Delta \varepsilon_{1}$, and hence $\Delta \varepsilon_{3}$ would be considerably smaller than $\Delta \varepsilon_{1}$. Total value of $\Delta \varepsilon_{1}$ and $\Delta \varepsilon_{3}$ is about 0.4 at the most and close to the value of $\Delta \varepsilon_{\alpha}$. In PET-I, the contribution of $\Delta \varepsilon_{3}$ to $\Delta \varepsilon_{\beta}$ is so small that the resolution of the $\beta$-peak would not be observed. Figure 15 indicates the relationship between $\Delta \varepsilon_{\beta}$ and $N_{3}$ at $0^{\circ} \mathrm{C} . \Delta \varepsilon_{\beta}$ increases slightly with an increase in 
$N_{3}$.

Resolution of double peaks in various samples is remarkable with an increase in the number of terminal carboxyl groups per unit volume. Activation energies for the $\beta_{1}$ and $\beta_{2}$ processes, $\Delta H_{1}{ }^{*}$ and $\Delta H_{2}{ }^{*}$, are 17 and $11 \mathrm{kcal} \mathrm{mol}^{-1}$, respectively.

Consequently, these facts lead us to the conclusion that the $\beta_{1}$-relaxation process is attributed to the local mode relaxation (model (1)) and the $\beta_{2}$-relaxation process to the rotational motion of terminal carboxyl groups. Therefore, effects of catalysts are scarcely recognized in the molecular structure of PET as a whole, but recognized in the local structure such as chain ends.

\section{CONCLUSION}

Among the samples used, only the noncatalyzed sample exhibited peculiar behavior electrically, i.e., the d.c. conductivity of this sample was quite small and resolution of the dielectric $\beta$-relaxation peak could not be observed. Effects of catalysts on d.c. conduction and on dielectric properties are different; the former is a direct effect and the latter is an indirect one. Consequently, we can conclude that residual catalysts are ionized in the polymer and contribute to an increase in d.c. conductivity.
Catalysts scarcely affect the molecular structure of PET as a whole, but would slightly alter local structures such as chain ends. We believe such investigations could contribute to the basis of material designs for polymers in electrical or electronic engineering.

\section{REFERENCES}

1. S. Saito, H. Sasabe, T. Nakajima, and K. Yada, J. Polym. Sci, Part A-2, 6, 1297 (1968).

2. I. M. Ward, Trans. Faraday Soc., 53, 1406 (1957).

3. S. Saito, "Researches of the Electrotechnical Laboratory", Electrotechnical Laboratory Ed., Sanpo-sha, Tokyo, 1964 No. 648.

4. S. Saito, Rep. Prog. Polym. Phys. Japan, 12, 407 (1969).

5. R. De P. Daubeny, C. W. Bunn, and C. J. Brown, Proc. Roy. Soc., Ser. A, 226, 531 (1954).

6. S. Saito and H. Sasabe, Rep. Prog. Polym. Phys. Japan, 12, 405 (1969); H. Sasabe and S. Saito, to be submitted to Polymer. $J$.

7. Yamafuji and Y. Ishida, Kolloid Z. Z. Polym. 183, 15 (1962).

8. K. H. Illers and H. Breuer, J. Colloid Sci., 18, 1 (1963).

9. K. Tajiri, Y. Fujii, M. Aida and H. Kawai, J. Macromol. Sci.-Phys. B4, 1 (1970).

10. Y. Ishida and K. Yamafuji, Kolloid Z. Z. Polym., 177, 97 (1961). 